Z Rheumatol $2017 \cdot 76: 474-476$

DOI 10.1007/s00393-017-0346-9

Online publiziert: 29. Juni 2017

c) Springer Medizin Verlag GmbH 2017

CrossMark

\section{U. Müller-Ladner}

Lehrstuhl für Innere Medizin mit Schwerpunkt Rheumatologie, Abteilung für Rheumatologie und Klinische Immunologie, Kerckhoff-Klinik GmbH, Justus-Liebig Universität Gießen, Bad Nauheim, Deutschland

\title{
Aktuelle Standards und neue Entwicklungen in der Psoriasisarthritis
}

Kaum eine andere Erkrankung unterliegt einer ähnlichen Dynamik hinsichtlich Entwicklungen in der Pathophysiologie und den hieraus resultierenden Therapieoptionen wie die Psoriasisarthritis. Aus diesem Grund hat sich für diese Kongress- bzw. Jubiläumsausgabe (90 Jahre Deutsche Gesellschaft für Rheumatologie [DGRh]) ein Expertenteam zusammengefunden, um Ihnen die Höhepunkte der aktuellen Aspekte rings um die Psoriasisarthritis im Detail vorzustellen.

\section{) Hinsichtlich Psoriasis/ -arthritis besteht eine enge Verknüpfung zwischen Rheuma- tologie und Dermatologie}

Schon der erste Beitrag von Herrn Prof. Pap als Rheumatologe und Herrn Prof. Sunderkötter als Dermatologe zeigt die enge Verknüpfung der beiden Fachgebiete, die gemeinsam die Parallelen, aber auch die Unterschiede zwischen Haut und Gelenken in den vergangenen Jahren erarbeitet haben. Der Hauptfokus der basiswissenschaftlichen Untersuchungen liegt hierbei v. a. auf den Signalwegen rings um Th17-Zellen und dem zugehörigen IL-17-Zytokin, wobei die mit IL17 eng verknüpften Zytokine IL-12 und IL-23 bei beiden Erkrankungsmanifestationen ebenfalls eine entscheidende Rolle spielen. Interessant ist bei diesem Komplex auch stets die Frage, ob die Psoriasis und die Psoriasisarthritis ein und dieselbe Erkrankung mit verschiedenen Ausprägungsformen darstellen oder es doch genetisch bedingte kli- nische Varianten außerhalb HLA-B27 gibt, die völlig getrennt ablaufen - dies auch vor dem Hintergrund, dass die Psoriasisarthritis trotz der bei der rheumatoiden Arthritis kaum vorhandenen proliferativen Komponente viele Überlappungen zur rheumatoiden Arthritis aufweist, wobei gerade das begrenzte Ansprechen der rheumatoiden Arthritis auf eine IL-17-Hemmung hier eine pathophysiologische Trennung begründen könnte.

Trotz der stets dermatologisch und rheumatologisch betonten Häufigkeit der Psoriasis/-arthritis in der Bevölkerung bleiben die Diagnose und Differenzialdiagnose in der Realität eine Herausforderung. Dies wird durch den Beitrag von Herrn Priv.-Doz. Dr. Gerdes und Herrn Prof. Mrowietz auf der dermatologischen und Herrn Prof. Wagner auf der rheumatologischen Seite sehr anschaulich illustriert. Dermatologisch wie rheumatologisch ist die erfolgreiche Suche nach den versteckten kutanen Läsionen als Schlüssel zur Diagnose sicher am häufigsten. Die nicht immer typischen Nagelveränderungen, die einer längeren antifungalen Medikation widerstehen, oder die minimalen proliferativen Gelenkveränderungen an den distalen Interphalangealgelenken als Ausdruck einer ,altersentsprechenden Fingerpolyarthrose" zeigen dann schon einen höheren Schwierigkeitsgrad. Klassisches Expertenwissen fordert zumeist die gering ausgeprägte Pustulosa oder die Differenzialdiagnose zu den entzündlichen Wirbelsäulenerkrankungen oder einem vertebral fokussierten Dish-Syndrom. Ebenso tückisch können sich die Enthesitiden und Seh- nenansatzverkalkungen vor dem Hintergrund entzündlich vs. degenerativ darstellen, sodass die Entscheidungstabelle der Autoren hierzu sicher hilfreiche Hinweise geben wird.

\section{\) Diagnose und Differen- zialdiagnose bei Psoriasis/- arthritis sind immer noch eine Herausforderung}

Im Spannungsfeld zwischen „in label“ und „off label“ sind Leitlinien und evidenzbasierte Therapieempfehlungen von Expertengruppen und Fachgesellschaften nicht nur Leitschiene für die täglichen Entscheidungen in Praxis und Klinik, sondern auch meist Rettungsanker für mehr oder weniger unangenehme Nachfragen der Kostenträger, insbesondere bei hochpreisigen Medikamenten und den regelhaft wiederkehrenden, zwar maßgeschneiderten, aber dennoch außerhalb der Zulassung liegenden Therapiestrategien für die individuellen Erkrankungskonstellationen. Da gerade bei der Psoriasis und Psoriasisarthritis verschiedene therapeutische Strategiekulturen bestehen, war es eine besondere Herausforderung für Frau Dr. Köhm und Herrn Dr. Behrens, diese in einem Beitrag gleichzeitig $\mathrm{zu}$ fusionieren und $\mathrm{zu}$ differenzieren. Grundlage aller dieser Strategien ist, - wie bei der für die rheumatoide Arthritis etablierten Treatto-target-Strategie - im Idealfall eine Remission, gleichbedeutend einer sichtund fühlbaren Ruhe der Grunderkrankung für Patient und Behandler, oder 
Hier steht eine Anzeige.




aber eine minimale Krankheitsaktivität, die sog. „minimal disease activity“ (MDA), schnellstmöglich zu erreichen. Mangels einer (noch) nicht verfügbaren deutschen Leitlinie können aktuell zum Management 2 internationale und vor Kurzem aktualisierte Therapieempfehlungen in der klinischen Routine herangezogen werden. Interessant ist hierbei, dass die Empfehlungen der European League Against Rheumatism (EULAR) und die der Group for Research and Assessment of Psoriasis and Psoriatic Arthritis (GRAPPA) unterschiedliche Herangehensweisen verfolgen. Die EULAR-Empfehlungen gehen wie bei der rheumatoiden Arthritis nach einem schrittweisen Therapiealgorithmus vor, der die Gesamterkrankung bzw. -aktivität im Fokus hat, während die Empfehlungen der GRAPPA den unterschiedlichen Manifestationsmustern jeweils bestimmte Medikamente zuordnet. Zudem unterscheidet die GRAPPA auch den Schweregrad der jeweiligen Manifestation, der durch die dermatologische und rheumatologische Expertengruppe durch Kriterien in mild, moderat und schwer eingeteilt wurden. Interessant ist auch, dass echte Head-to-head-Studien lediglich für die Therapie der Psoriasis zur Verfügung stehen.

Natürlich wird regelmäßig die Frage gestellt, welche der vielen neuen Therapiekonzepte und Medikamente dann baldmöglichst den Weg in die Klinik finden. Frau Dr. Danzer und Herr Prof. Fleck erläutern in ihrem Beitrag, welche Optionen in der Zukunft realisiert werden können und welche unter Umständen noch etwas mehr Zeit benötigen. Hauptproblem ist wie immer die ausgeprägte Variabilität, nicht nur innerhalb der „Hautvarianten“, auch die Vielfalt der Gelenkmanifestationen macht eine zielgerichtete Medikamentenentwicklung nicht gerade einfacher. Dazu kommt, dass sich die Erscheinungsbilder über die Zeit und durch die bereits angewandten Medikamente verändern und sich wie in der Infektiologie ,immunologische Resistenzen“" entwickeln können, selbst wenn diese nur temporär bestehen. Neben den vielfältigen Ansätzen neuer Biologika, die wie Brodalumab, Bimekizumab und ABT-122 hauptsäch- lich die pathophysiologisch aktuellen IL-17 (IL-12/23)-assoziierten Moleküle zum Ziel haben, könnten aber auch wie bei der rheumatoiden Arthritis die sog. „targeted small molecules“, v. a. die Januskinaseinhibitoren Tofacitinib und Baricitinib, aber auch Sphingolipidagonisten wie Ponesimod und der A3ARAgonist CF101 zukünftig eine tragende Rolle bei der Behandlung der Psoriasis/ -arthritis spielen. Es bleibt zu hoffen, dass für diese neuen Medikamente stets genügend Patienten für die Rekrutierung in die Zulassungsstudien zur Verfügung stehen.

Viel Spaß beim Lesen dieses ganz speziellen Schuppenflechten-Gelenkentzündungshefts und natürlich bei der Diskussion dieser Ideen und Ansätze mit den Kollegen beim DGRh 2017 in Stuttgart.

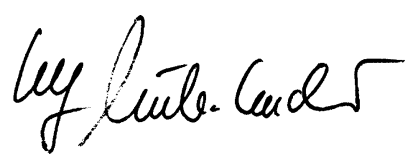

U. Müller-Ladner

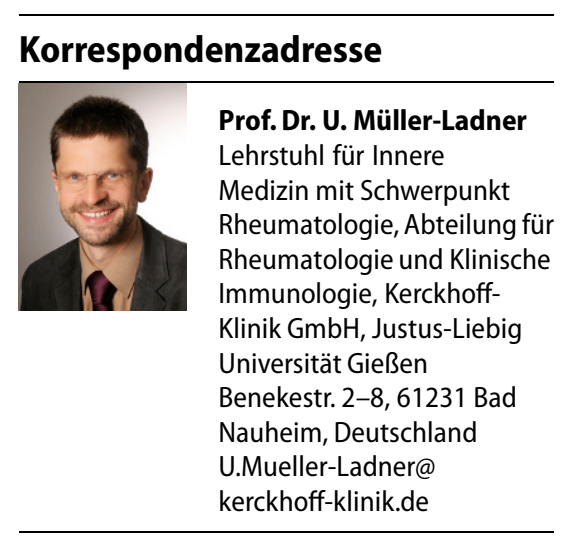

Interessenkonflikt. U. Müller-Ladner gibt an, dass kein Interessenkonflikt besteht.

\section{Rheuma geht aufs Herz}

„An Gelenkschmerzen ist noch niemand gestorben - aber sehr wohl an rheumatischen Herzschäden"

Eine mehrjährige Studie am Stuttgarter Robert-Bosch-Krankenhaus zeigt: Rheuma erhöht das Risiko für Herzerkrankungen. Eine angepasste Therapie kann dem Tod durch Herzschäden bei Rheumapatienten vorbeugen.

Die Ärzte kamen in der mehrjährigen Studie zu neuen Erkenntnissen über die Auswirkungen von Rheumaerkrankungen auf das Herz. In einer ersten Studienstufe untersuchten und beobachteten sie rund 300 Rheumapatienten. Das Ergebnis: Art und Häufigkeit der Herzbeteiligung sind abhängig von der Art der rheumatischen Erkrankung. Die Häufigkeit variiert von eher selten bis häufig. Bei nur 14 Prozent der Patienten mit rheumathoider Arthritis ist das Herz beteiligt, aber 54 Prozent der Patienten mit Gefäßentzündungen, der sogenannten ANCA-assoziierten Vaskulitis, leiden an rheumatischen Herzschäden.

Krankheitsspezifische Narbenmuster Je nach Rheumaerkrankung entstehen unterschiedliche, aber krankheitsspezifische Narbenmuster, die in der MRT-Bildgebung sichtbar werden. „Diese Narbenmuster unterscheiden sich deutlich von denen, die beispielsweise bei einem Herzinfarkt oder einer vererbten Herzmuskelerkrankung entstehen", erklärt Professor Mahrholdt. So lässt sich erkennen, ob vom Rheuma auch das Herz betroffen ist. Ist dies der Fall, ist eine Anpassung der Therapie erforderlich.

Die Studiengruppe nahm in einem zweiten Studienschritt eine neuartige Form der MRT-Technik, das sogenannte MyokardMapping, zu Hilfe. Sie untersuchte damit Rheumapatienten mit und ohne Herznarben. Es zeigte sich, dass mit dieser verfeinerten Bildgebung Herzerkrankungen bereits vor der Narbenbildung diagnostiziert werden können.

Quelle: Marlies Kepp, Robert-Bosch-Krankenhaus Stuttgart 